This item was submitted to Loughborough's Research Repository by the author.

Items in Figshare are protected by copyright, with all rights reserved, unless otherwise indicated.

\title{
Trapping structures in the three-dimensional water-wave problem
}

PLEASE CITE THE PUBLISHED VERSION

PUBLISHER

(c) Cambridge University Press

LICENCE

CC BY-NC-ND 4.0

\section{REPOSITORY RECORD}

Mclver, P., and J.N. Newman. 2019. "Trapping Structures in the Three-dimensional Water-wave Problem". figshare. https://hdl.handle.net/2134/2218. 


\title{
Trapping structures in the three-dimensional water-wave problem
}

\author{
By P. MCIVER ${ }^{1}$ AND J. N. NEWMAN ${ }^{2}$ \\ ${ }^{1}$ Department of Mathematical Sciences, \\ Loughborough University, Loughborough, Leicestershire LE11 3TU, UK \\ ${ }^{2}$ Department of Ocean Engineering, Massachusetts Institute of Technology, \\ Cambridge, MA 02139, USA
}

(Received 31 October 2002)

Trapped modes in the linearized water-wave problem are free oscillations of the fluid which have finite energy. They are known to exist at isolated frequencies in the presence of certain special structures. The existence of a trapped mode implies the non-uniqueness, or non-existence, of the solution to physically relevant radiation and diffraction problems for such a structure.

Previous work on the three-dimensional problem has established the existence of vertically axisymmetric structures that support trapped modes with either a single interior free surface, or two concentric interior free surfaces. In the present work the existence of several new types of trapping structures is established. These include non-axisymmetric structures with a single interior free surface and various structures with multiple interior free surfaces. The method used is an indirect one in which flow fields without wave radiation are specified, and corresponding structures are found by constructing suitable stream surfaces. Computations of the added-mass coefficients for these structures provide independent support for the existence of a trapping mode and illustrate their hydrodynamic characteristics at other wavenumbers.

\section{Introduction}

Trapped modes are free oscillations with finite energy of an unbounded fluid with a free surface for which the fluid motion is essentially confined to the vicinity of a fixed structure. More specifically, in the linearized water-wave problem they are non-trivial solutions that satisfy homogeneous boundary conditions and for which there are no waves in the far field, and hence there is an absence of any forcing either from incident waves or from an imposed motion of the structure. Until quite recently it was unknown whether or not such trapped modes exist. Indeed, the classical uniqueness proof of John (1950) and subsequent extensions show that trapped modes cannot exist for wide classes of floating and submerged bodies. However, it is now established that such modes do indeed exist and may be supported at specific frequencies by certain 'trapping structures' of finite extent. Surface-piercing and submerged trapping structures have been constructed in both the two- and three-dimensional water-wave problems (see McIver \& Porter 2002, and the references therein). The existence of a trapped mode at a particular frequency implies the non-uniqueness, or even nonexistence, of the solution to the frequency-domain scattering or radiation problem at that frequency (McIver 1997). 

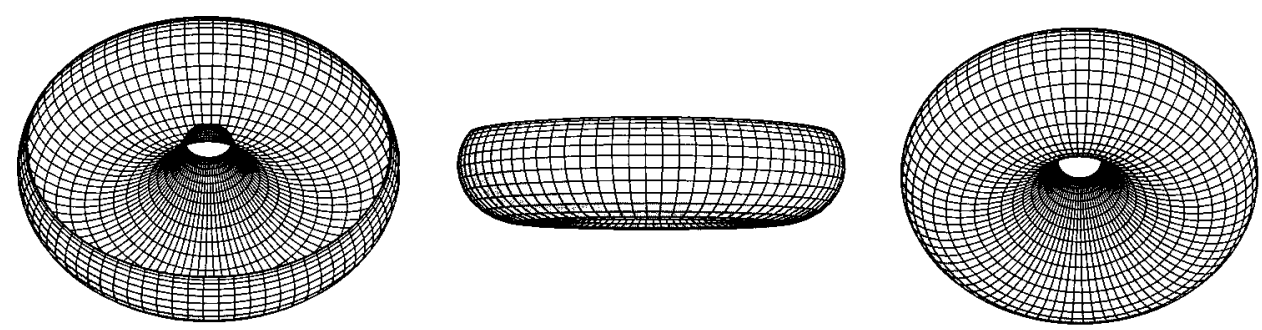

FIGURE 1. Three views of the submerged surface of an axisymmetric trapping structure generated from a ring source with radius $c=j_{0,1} / K$. The left-hand view is looking down from a point above the free surface, the middle view is from a submerged point alongside the structure, and the right-hand view is from a more deeply submerged point.

Two different procedures have been followed to search for examples of trapping structures. In the 'indirect' approach a flow field is constructed analytically from appropriate combinations of time-harmonic singularities in such a way that their radiated waves are completely cancelled in the far field. A numerical procedure is then used to obtain the streamlines or stream surfaces generated by these singularities; any of these lines or surfaces may be used to define the geometry of a trapping structure as long as there are no paths within the fluid that join a singularity to infinity. In the 'direct' approach a family of body shapes is considered. These shapes are usually of simple form so that they may be described by a small set of geometrical parameters; suitable conditions are imposed to specify a homogeneous solution of the boundary-value problem, and a numerical search for non-trivial solutions is made within the parameter space.

Examples of axisymmetric trapped modes in the presence of axisymmetric structures were constructed by McIver \& McIver (1997) using the indirect procedure, with the flow field generated by a time-harmonic circular ring source of radius $c$ in the plane of the free surface. In this case the amplitude of the radiated waves in the far field is proportional to $J_{0}(K c)$; here $J_{0}$ is the Bessel function of order zero and the wavenumber $K=\omega^{2} / g$, where $\omega$ is the radian frequency and $g$ is the acceleration due to gravity. Thus there is no wave radiation at the frequencies given by $K c=j_{0, n}$, where $j_{0, n}$ is the $n$th zero of the Bessel function $J_{0}$. Suitably chosen axisymmetric stream surfaces of this flow generate a family of toroidal structures intersecting the free surface which, by construction, are able to support free oscillations of the fluid; one example of such a structure is shown in figure 1 for the case $n=1$. The stream surfaces exclude the singularity from the flow field and the motion decays sufficiently quickly with distance for the total energy of the fluid motion to be finite. The construction of McIver \& McIver (1997) was extended by Kuznetsov \& McIver (1997) to give non-axisymmetric trapped modes in the presence of axisymmetric toroidal structures by allowing the strength of the ring source, and hence the corresponding velocity field, to have a sinusoidal azimuthal variation.

Newman (1999) performed a hydrodynamic analysis of surface-piercing axisymmetric trapping structures using the radiation/diffraction code WAMIT. When the added mass and damping (divided by $\omega$ ) are regarded as the real and imaginary parts of a function of the complex frequency $\omega$, a trapped mode corresponds to a pole of this function on the positive real $\omega$ axis. Thus, in numerical calculations, singular behaviour of the added mass and damping is observed in the vicinity of the trappedmode frequency and the existence of a trapped mode can have important practical consequences. Conversely, the observed presence of these singularities in computations 
of the added mass and damping provides strong supporting evidence for the existence of trapped modes. In the limit as a discretized structure approaches the trapping structure, the singularity of the added mass coefficient is proportional to $\left(\omega-\omega_{0}\right)^{-1}$, where $\omega_{0}$ is the frequency of the trapped mode, whereas the singularity of the damping coefficient is proportional to the delta-function $\delta\left(\omega-\omega_{0}\right)$. In computations with a high but finite level of precision it is very difficult to detect the latter singularity. For that reason the computational results given below for the new trapping structures refer only to the added mass.

Originally the direct approach was used only for infinite periodic arrays of structures, or equivalently for structures within channels, as for example in the work of Callan, Linton \& Evans (1991) and Evans \& Porter (1998). More recently this approach has been used to derive axisymmetric trapping structures by McIver \& Porter (2002) and Shipway \& Evans (2002). McIver \& Porter (2002) establish the existence of submerged trapping structures of toroidal form with elliptical sections. In the work of Shipway \& Evans (2002) two concentric surface-piercing circular cylinders are used, with vertical axis and zero thickness; for this example there are two interior free surfaces, one within the inner cylinder and the other in the annulus between the two cylinders. Several different mode shapes are found, corresponding to different combinations of the geometric parameters. In all of their examples the elevation of the inner free surface contains at least one nodal circle; no solutions were derived that are without free-surface nodes, corresponding to so-called 'pumping' motions in both of the interior free-surface portions. These pumping modes occur at relatively low frequencies, and are of the greatest practical concern for offshore structures with interior 'moonpools'.

The present work is based on more extensive use of the indirect approach and demonstrates the existence of several new types of trapping structure. These include structures which are not axisymmetric, with a single inner free surface, and structures for which there are two separate inner free surfaces. In the former case, both axisymmetric and non-axisymmetric arrangements of singularities are employed. In the latter case we show examples where there are no nodes within either interior free surface. The demonstrations of the existence of non-axisymmetric trapping structures with an internal free surface suggest that it may be possible for some offshore structures (for example, a barge with a moon pool) to support trapped modes.

\section{The boundary-value problem}

The fluid is assumed to have infinite depth and to be inviscid and incompressible, and the motion is assumed to be irrotational. Rectangular Cartesian coordinates $(x, y, z)$ are chosen so that the mean free surface coincides with the plane $z=0$ and the $z$-axis is directed vertically downwards. For time-harmonic motions of angular frequency $\omega$, the flow may be described by a velocity potential

$$
\Phi(\boldsymbol{x}, t)=\operatorname{Re}\left\{\phi(\boldsymbol{x}) \mathrm{e}^{\mathrm{i} \omega \mathrm{t}}\right\}
$$

where $t$ is time and the potential $\phi$ is a function of position $\boldsymbol{x}$ only. In general this function is complex, but in the context of trapped modes where the boundary conditions are homogeneous and no propagating or radiating waves are present in the far field, $\phi$ may be assumed to be real without loss of generality. 
The potential $\phi$ of a trapped mode in the presence of a structure is a non-trivial solution of

$$
\begin{gathered}
\nabla^{2} \phi=0 \quad \text { in the fluid } W \\
\frac{\partial \phi}{\partial n}=0 \quad \text { on the surface of the structure } S \\
\frac{\partial \phi}{\partial z}+K \phi=0 \quad \text { on the free surface } F
\end{gathered}
$$

such that

$$
\int_{W}|\nabla \phi|^{2} \mathrm{~d} x \mathrm{~d} y \mathrm{~d} z+K \int_{F}|\phi|^{2} \mathrm{~d} x \mathrm{~d} y<\infty .
$$

Here $K=\omega^{2} / g$ is the wavenumber of a plane wave with the same frequency. The first and second integrals in (2.5) are proportional to the kinetic and potential energies, respectively. Thus (2.5) is equivalent to the statement that $\phi$ and $\nabla \phi$ decay sufficiently rapidly at infinity for the fluid motion to have finite energy.

This is an eigenvalue problem with $K$ as the spectral parameter. The main object of the present paper is to determine structural geometries, and the corresponding values of $K$, for which non-trivial solutions for $\phi$ exist in the above problem.

\section{Construction of trapping structures}

For all of the examples of trapping structures described here, a flow field is first specified in terms of one or more rings of wave sources and then suitable stream surfaces are formed by tracking individual streamlines. Given a flow field with potential $\phi$, streamlines can be obtained by the simultaneous numerical integration of

$$
\frac{\mathrm{d} x}{\mathrm{~d} \tau}=\frac{\partial \phi}{\partial x}, \quad \frac{\mathrm{d} y}{\mathrm{~d} \tau}=\frac{\partial \phi}{\partial y}, \quad \frac{\mathrm{d} z}{\mathrm{~d} \tau}=\frac{\partial \phi}{\partial z},
$$

where $\tau$ is a parameter that increases monotonically along the streamline (Pozrikidis 1997, §1.7). This method has the virtue of being very versatile and is used for a few streamlines in the examples below. However, in the constructions here most streamlines of interest begin and end on the free surface and the above method has the disadvantage that the range of $\tau$ required to achieve this is a priori unknown. In most of the examples below it is convenient to exploit the geometry and to work in terms of toroidal coordinates $(r, \theta, \beta)$ with $r>0,0 \leqslant \theta<\pi$, and $0 \leqslant \beta<2 \pi$ (Bourne $\&$ Kendall 1977), which are related to the Cartesian coordinates by

$$
x=(c-r \cos \theta) \cos \beta, \quad y=(c-r \cos \theta) \sin \beta, \quad z=r \sin \theta,
$$

where $c$ is the radius of a circular ring in the free surface (perhaps, but not necessarily, coinciding with a ring source as in $\S 1$ ). Thus, $\beta$ is an azimuthal angle measured around the $z$-axis and, in any vertical plane through the $z$-axis, $(r, \theta)$ are equivalent to plane polar coordinates with origin at $R=\left(x^{2}+y^{2}\right)^{1 / 2}=c, z=0$. (Here the origin $O_{\mathrm{t}}$ of the toroidal-coordinate system is taken to coincide with the origin $O$ of the Cartesian coordinates; this need not be the case and it is often convenient to displace $O_{\mathrm{t}}$ relative to $O$.) To track a streamline that leaves and enters the free surface $z=0$ on either side of $R=c$ it is sufficient to integrate the equations in $\theta$ given below from $\theta=0$ to $\theta=\pi$ (or vice versa). 
Let $\boldsymbol{e}_{r}, \boldsymbol{e}_{\theta}$, and $\boldsymbol{e}_{\beta}$ be unit vectors in the $r$-, $\theta$ - and $\beta$-directions respectively. With the time dependence removed as in (2.1), the velocity is

$$
\nabla \phi=\frac{\partial \phi}{\partial r} \boldsymbol{e}_{r}+\frac{1}{r} \frac{\partial \phi}{\partial \theta} \boldsymbol{e}_{\theta}+\frac{1}{c-r \cos \theta} \frac{\partial \phi}{\partial \beta} \boldsymbol{e}_{\beta},
$$

and it is required to determine a surface $r=r(\theta, \beta)$ such that

$$
\nabla \phi \cdot \boldsymbol{n}=0,
$$

for all normals

$$
\boldsymbol{n}=-\boldsymbol{e}_{r}+\frac{1}{r} \frac{\partial r}{\partial \theta} \boldsymbol{e}_{\theta}+\frac{1}{c-r \cos \theta} \frac{\partial r}{\partial \beta} \boldsymbol{e}_{\beta}
$$

to the surface. In other words, the kinematic condition to be satisfied on a structural surface is

$$
q_{\theta} \frac{\partial r}{\partial \theta}+q_{\beta} \frac{\partial r}{\partial \beta}=q_{r}
$$

where

$$
q_{r}=\frac{\partial \phi}{\partial r}, \quad q_{\theta}=\frac{1}{r^{2}} \frac{\partial \phi}{\partial \theta}, \quad q_{\beta}=\frac{1}{(c-r \cos \theta)^{2}} \frac{\partial \phi}{\partial \beta} .
$$

Now for $q_{\theta} \neq 0$ equation (3.6) can be rewritten as

$$
\frac{\partial r}{\partial \theta}+\frac{q_{\beta}}{q_{\theta}} \frac{\partial r}{\partial \beta}=\frac{q_{r}}{q_{\theta}}
$$

so that

$$
\frac{\mathrm{d} r}{\mathrm{~d} \theta}=\frac{q_{r}}{q_{\theta}}
$$

on the curves

$$
\frac{\mathrm{d} \beta}{\mathrm{d} \theta}=\frac{q_{\beta}}{q_{\theta}} .
$$

The last two equations determine the streamlines of the flow which are just the characteristic curves for the partial differential equation (3.6); see for example Courant \& Hilbert (1962, Chapter II, §1). Under certain not very restrictive conditions, given an initial curve $\Gamma$ in the free surface defined by $r=r(0, \beta)$, equations (3.9)-(3.10) can be integrated from initial points $\left(r\left(0, \beta_{0}\right), 0, \beta_{0}\right)$ on $\Gamma$ to determine curves that are everywhere tangent to the velocity field. Alternatively, an initial curve $\Gamma$ defined by $r=r(\pi, \beta)$ may be chosen and equations (3.9)-(3.10) then integrated from initial points $\left(r\left(\pi, \beta_{0}\right), \pi, \beta_{0}\right)$. Thus, given the velocity field and an appropriately chosen initial closed curve $\Gamma$ in the free surface, a stream surface can be generated by simultaneous integration over $0 \leqslant \theta \leqslant \pi$ of the two first-order differential equations (3.9)-(3.10). Since the singularity in the free surface at $R=c$ must be totally enclosed by the structure, the curve $\Gamma$ is situated entirely interior or entirely exterior to $R=c$.

For the special case in which the specified flow is axisymmetric, so that $q_{\beta}=0$, equation (3.10) gives immediately that $\beta$ is constant on any characteristic curve and it is sufficient to integrate (3.9) to determine the streamlines. Although the velocity field is axisymmetric there is no requirement that the initial curve $\Gamma$ must also be axisymmetric. Thus, non-axisymmetric stream surfaces can be generated from axisymmetric velocity fields.

If the $\beta$-dependence in the velocity field is separable so that

$$
q_{r}=Q_{r}(r, \theta) \cos m \beta, \quad q_{\theta}=Q_{\theta}(r, \theta) \cos m \beta, \quad q_{\beta}=Q_{\beta}(r, \theta) \cos m \beta,
$$


where $m$ is an integer, then the right-hand side of (3.9) is independent of $\beta$ and again the $r$-integration can be performed separately. In previous work by Kuznetsov \& McIver (1997) initial curves $\Gamma$ were chosen as circles centred on $R=0$ so that the stream surfaces generated are axisymmetric, although the flow field itself is not axisymmetric. It is possible to generate non-axisymmetric trapping structures from flow fields of this type, but they are not described here.

Another approach to the axisymmetric flow case is to express the given velocity field in terms of a function $\psi(r, \theta, \beta)$ so that

$$
\nabla \phi=-\frac{1}{r(c-r \cos \theta)} \frac{\partial \psi}{\partial \theta} \boldsymbol{e}_{r}+\frac{1}{r(c-r \cos \theta)} \frac{\partial \psi}{\partial r} \boldsymbol{e}_{\theta} ;
$$

the particular form arises from the requirement that $\nabla^{2} \phi=0$. For some constant $C$, a normal to a surface $S$ defined by

$$
\psi(r, \theta, \beta)=C
$$

is

$$
\nabla \psi=\frac{\partial \psi}{\partial r} \boldsymbol{e}_{r}+\frac{1}{r} \frac{\partial \psi}{\partial \theta} \boldsymbol{e}_{\theta}+\frac{1}{c-r \cos \theta} \frac{\partial \psi}{\partial \beta} \boldsymbol{e}_{\beta}
$$

and the construction ensures that $\nabla \psi$ is perpendicular to $\nabla \phi$ everywhere on $S$, and hence $S$ is a stream surface of the flow. Given an axisymmetric velocity field, (3.12) can be solved to determine $\psi$; this is probably best done in a different coordinate system. In most applications $\psi$ is chosen to be axisymmetric (and often called the Stokes stream function) so that $\psi$ is independent of $\beta$ and the surfaces $S$ are also axisymmetric. This is the approach to this problem initially adopted by McIver \& McIver (1997) in order to generate axisymmetric trapping structures from axisymmetric velocity fields. However, if for a given $\phi$

$$
\psi(r, \theta, \beta)=\Psi(r, \theta)
$$

is a particular solution to (3.12) then

$$
\psi(r, \theta, \beta)=\Psi(r, \theta)+\chi(\beta)
$$

is also a solution for any reasonable $\chi(\beta)$. In general, the surfaces $S$ generated from (3.13) using (3.16) are not axisymmetric.

\section{Axisymmetric flow fields}

In this section examples are given of non-axisymmetric trapping structures that are obtained from the stream surfaces of axisymmetric flow fields. Many types of non-axisymmetric trapping structures can be generated by the characteristic method described in $\S 3$. For instance, the structure shown in figure 1 can be distorted so that the inner and outer radii both vary with the azimuthal angle $\beta$ simply by choosing a non-circular initial curve $\Gamma$. A little care must be taken in the choice of $\Gamma$ as there is a discontinuity in the stream function at the ring source; this is illustrated in figure 2 for ring sources with $(a) K c=j_{0,1}$, and $(b) K c=j_{0,2}$. The Stokes stream function is given by equations (13)-(14) of McIver \& McIver (1997) and the streamline patterns for both of these cases are given in that paper. However, for later convenience streamlines for case $(b)$ are shown here in figure 3. Stream surfaces that form trapping structures are formed from streamlines that each begin and end on the free surface and join points with the same value of the stream function $\psi$. It is essential that there is a stream surface that excludes the singularity from the flow field. 

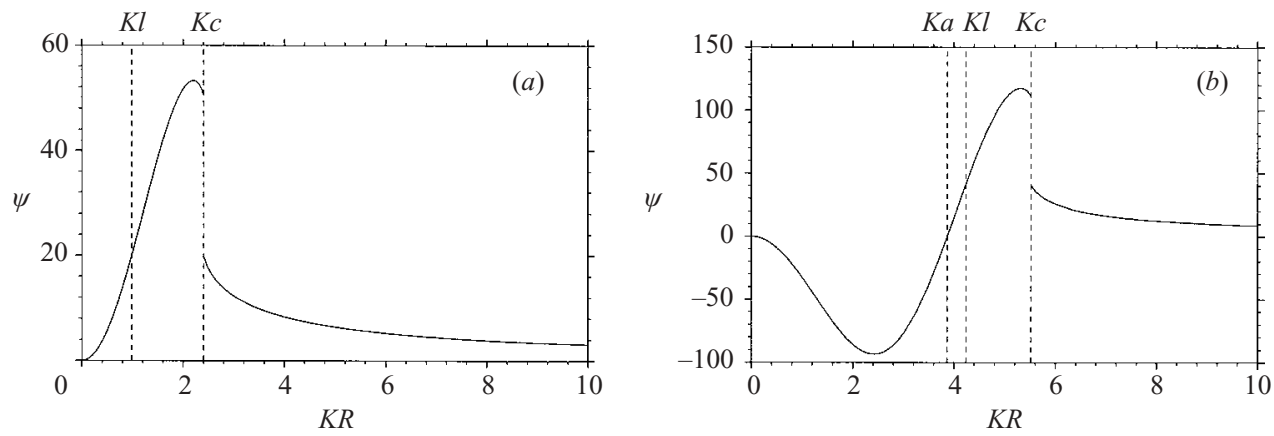

FiguRE 2. Free-surface variation of the stream function $\psi$ for a ring source of radius $c$ as a function of the radial distance $R$. Streamlines with a free-surface intersection point in the range $l \leqslant R<c$ enter the source directly from the fluid region. (a) $K c=j_{0,1} \approx 2.4048$, $K l \approx 0.9846$. (b) $K c=j_{0,2} \approx 5.5201, K l \approx 4.2357$. In case $(b)$, streamlines exist with two free-surface intersections in the range $0<R<a$ where $K a \approx 3.8685$.

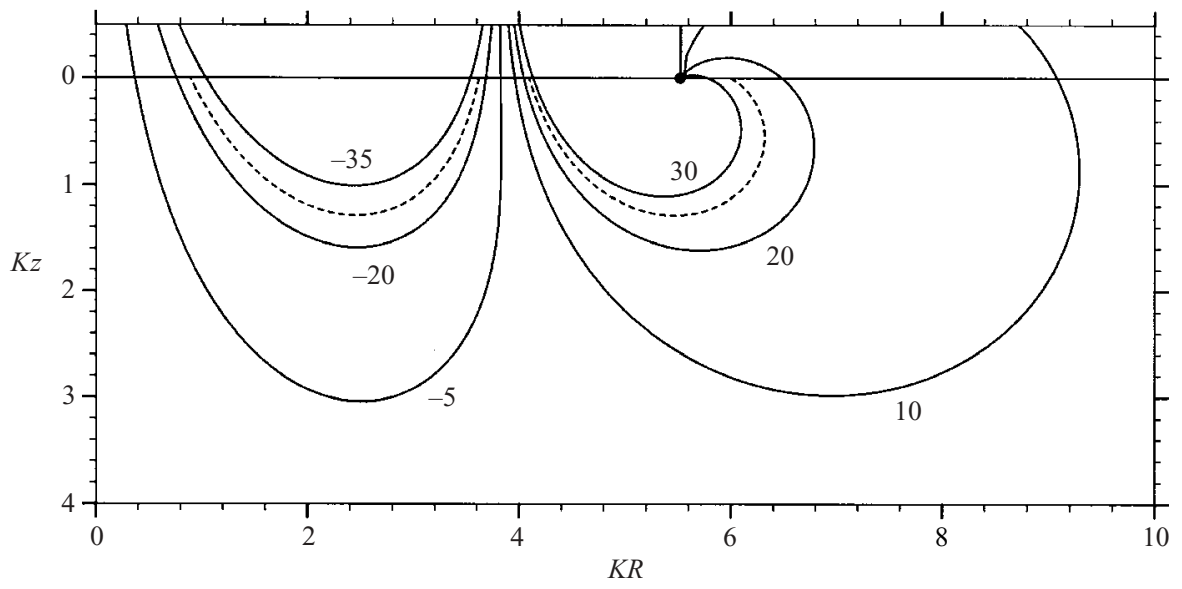

FIGURE 3. Streamline pattern for a ring source placed at $(R, z)=(c, 0)$, where $K c=j_{0,2}$. The solid streamlines are marked with the corresponding values of the stream function, while the dashed streamlines are used for the computations of the added mass shown in figure 8 . The position of the ring source is shown by a filled circle.

In both of the cases illustrated in figure 2, all values of $\psi$ on the free surface for $R>c$ also occur for $0<R<c$; thus all streamlines emanating from points exterior to the ring source surround the singularity and any choice of $\Gamma$ exterior to the ring source is valid. Streamlines that leave the free surface in $l \leqslant R<c$, where $\psi(l)=\psi\left(c_{+}\right)$, subsequently enter the singularity directly from the fluid and cannot be part of a trapping structure; hence, any choice of $\Gamma$ interior to the ring source must lie in $0<R<l$. In case $(a)$ any streamline emanating from the free surface in this interval will return to the free surface outside the ring source. In case $(b)$ any streamlines starting from a point in $a<R<l$, where $\psi(a)=0$, return to the free surface outside $R=c$, but streamlines starting from a point in $0<R<a$ return to the free surface within the same interval; examples of these inner streamlines, corresponding to negative values of $\psi$, are shown in figure 3 . Thus, in case $(b)$, any trapping structure must include an outer element which coincides with one of the streamlines $\psi>0$, so that the singularity is excluded from the flow field, but there 


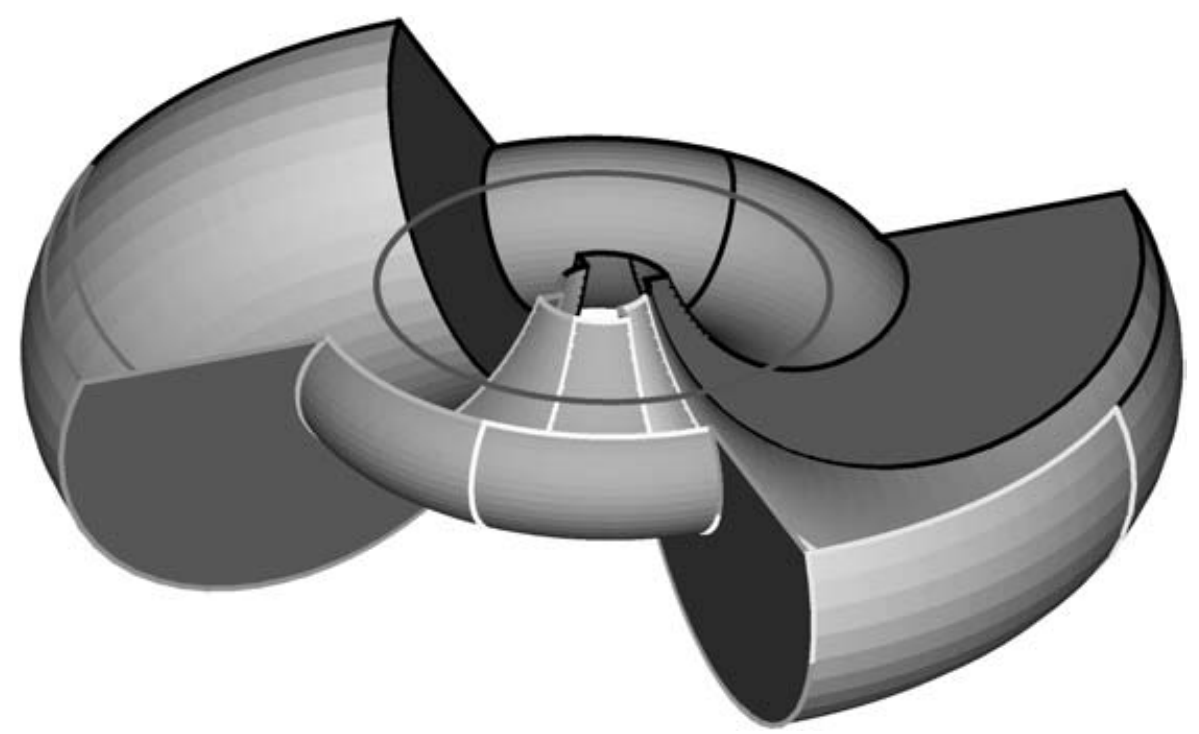

FIGURE 4. Perspective view of a non-axisymmetric trapping structure constructed from sections of different radius. The dark lines show the boundaries of each patch and its reflections about the planes of symmetry. The position of the ring source at $c=j_{0,1}$ is also shown.

is the option of including an additional element which coincides with one of the streamlines $\psi<0$.

The value of the parameter $K c$ specified in the construction may be interpreted in two ways. For a fixed geometry it defines the corresponding value of the frequency parameter, or eigenvalue, $K$. On the other hand, $K$ may be specified and it is then possible to find a family of trapping structures corresponding to the ring radius $c$. For most of the results in this paper the interpretation is left open. For numerical calculations of hydrodynamic quantities where a specific choice must be made, the trapped-mode frequency is fixed at $K=1$.

The example of a non-axisymmetric trapping structure shown in figure 4 is constructed by joining sections of two different axisymmetric trapping structures, both of which are generated by the same ring source of radius $c=j_{0,1}$ (so that the trapped-mode frequency corresponds to $K=1$ ). These are joined by azimuthal planes, which also are stream surfaces. To provide independent evidence that this is indeed a trapping structure, the added mass coefficient for this geometry has been computed as a function of frequency using the program WAMIT. As noted in the introduction, for any trapping structure there will be singular behaviour of the added mass at the trapped-mode frequency.

For these computations a higher-order method is used, with quadratic B-spline representation of the potential and 'exact' representation of the geometry. For this purpose the geometry of the structure shown in figure 4 is defined explicitly following the method described by Lee \& Newman (2001). Three 'patches' are defined in one quadrant as follows. Patch 1 consists of a partial torus with inner waterline radius $0.2 c$ restricted to the range $\beta \in(0, \pi / 4)$, patch 2 consists of a partial torus with inner waterline radius $0.3 c$ restricted to the range $\beta \in(\pi / 4, \pi / 2)$, and patch 3 is the portion of the azimuthal plane $\beta=\pi / 4$ between the generating sections of the first two patches. After reflection about the planes $x=0$ and $y=0$ a non-axisymmetric closed structure is formed, with the property that its surface coincides with the axisymmetric 


\begin{tabular}{rcll}
$N$ & Elements & \multicolumn{1}{c}{$K_{0}$} & $\max \left(\left|a_{33}\right|\right)$ \\
48 & $2 \times 2$ & 1.011 & $4.5 \times 10^{2}$ \\
108 & $4 \times 4$ & 1.0007 & $6.6 \times 10^{4}$ \\
300 & $8 \times 8$ & 1.000005 & $2.4 \times 10^{6}$
\end{tabular}

TABLE 1. Value of the singular wave number $K_{0}$ and the maximum absolute value of the added mass $a_{33}$, as functions of the number of unknowns $N$.

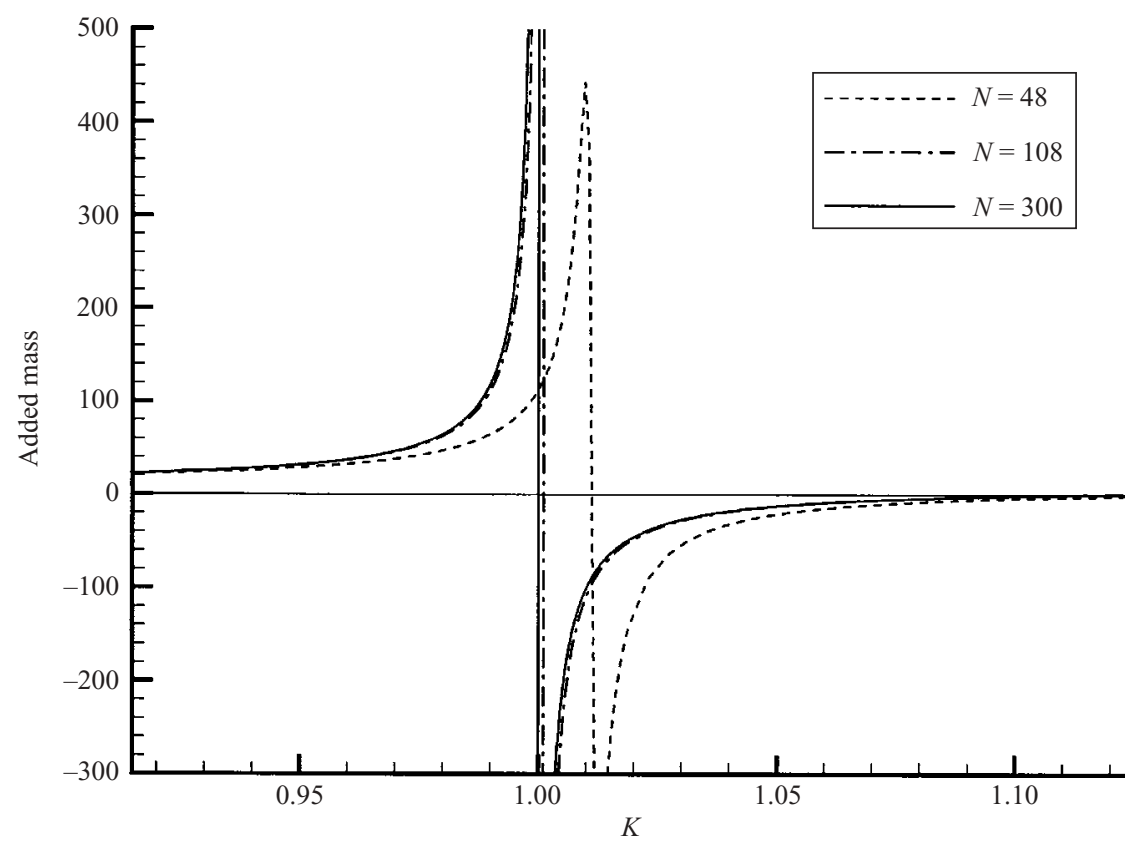

FIGURE 5. Heave added-mass coefficient of the structure shown in figure 4, normalized with respect to the radius of the ring source and fluid density, vs. the wavenumber $K$.

stream surfaces generated by the ring source. The toroidal radial coordinates of the generating sections for the first two patches are defined by economized polynomials of degree 10 in the angle $\theta$. The maximum error in these polynomial approximations is $5 \times 10^{-6}$.

Figure 5 shows the heave added-mass coefficient for this structure in the vicinity of the trapped wavenumber. The accuracy of these computations is refined by increasing the number of control points (knots) for the B-spline representation of the velocity potential. Three different curves are shown, with $N=48,108$, and 300 unknowns in the linear system of equations, corresponding to subdivisions of each patch into $2 \times 2,4 \times 4$, and $8 \times 8$ elements. These results are computed in the range shown using a large number of closely spaced wavenumbers. In the vicinity of the singular wavenumber $K=1$ the increment required to identify the peaks and critical value of $K_{0}$ is as small as $\Delta K=10^{-6}$. The value $K_{0}$ of the wavenumber where the added-mass coefficient changes sign, and the maximum value of this coefficient, are shown in table 1 . As the computational accuracy is refined, the maximum value of the addedmass coefficient increases without apparent limit, and the critical wavenumber tends 

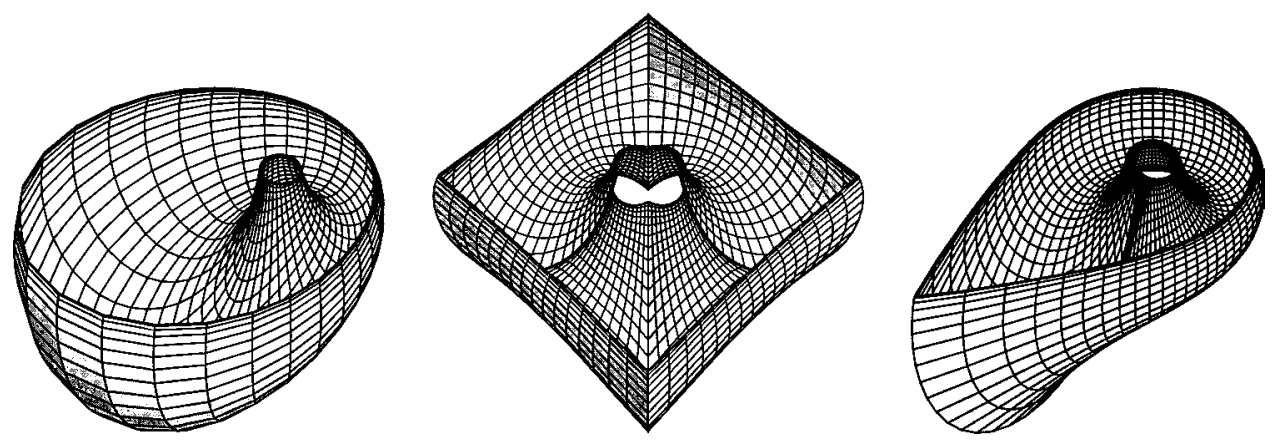

FIGURE 6. Perspective views of the submerged surfaces of some non-axisymmetric trapping structures generated from a ring source at $c=j_{0,1} / K$.

toward the theoretical value $K=1$. These numerical results give strong supporting evidence for the existence of non-axisymmetric trapping structures.

Some other examples of non-axisymmetric trapping structures are shown in figure 6 for both smooth and non-smooth initial curves. In each case the surfaces were generated from a single ring source with $K c=j_{0,1}$ by choosing points on $\Gamma$, and then integrating equations (3.9)-(3.10) from each of these points until the free surface is met on the other side of the ring source. The lines that join the inner and outer free surfaces are the computed streamlines; other lines are introduced to help visualize the surface. Although two of the examples of trapping structures shown in figure 6 have edges where there is a discontinuity in the surface slope, there is no corresponding singularity in the velocity field as the construction ensures that the flow is always along, rather than around, each edge. Computations of the heave added mass have been made for the structures illustrated in figure 6 with very similar results to those already shown in figure 5 .

\section{Concentric axisymmetric structures}

In this section we describe trapping structures that are obtained from the stream surfaces of the axisymmetric flow field formed by one ring source, of radius $c=j_{0, n} / K$. When $n>1$ it is possible to have up to $n$ separate elements of the structure, with the same number of moonpools. For simplicity we restrict our consideration to the case where all the elements are axisymmetric and concentric, but it would be straightforward to consider non-axisymmetric elements in the same manner as in the previous section.

Shipway \& Evans (2002) have demonstrated the existence of axisymmetric trapping structures formed from two concentric surface-piercing cylinders with zero thickness. Analogous trapping structures with finite volumes may also be found by the present method using a ring source at $c=j_{0, n} / K$, where $n>1$. For increasing $n$ the number of nodes on the interior free surface increases, and the results presented by Shipway \& Evans (2002) include one or more nodes, corresponding to $n \geqslant 3$. In order to derive analogous results without nodes on the free surface we use $n=2$ and select the streamlines $\psi= \pm 20$ from those shown in figure 3 . The corresponding free-surface potential, which is proportional to the free-surface elevation, is shown in figure 4 of McIver \& McIver (1997) and it can be seen that the fluid oscillations in the two enclosed portions of the free surface are out-of-phase 'pumping' motions. 


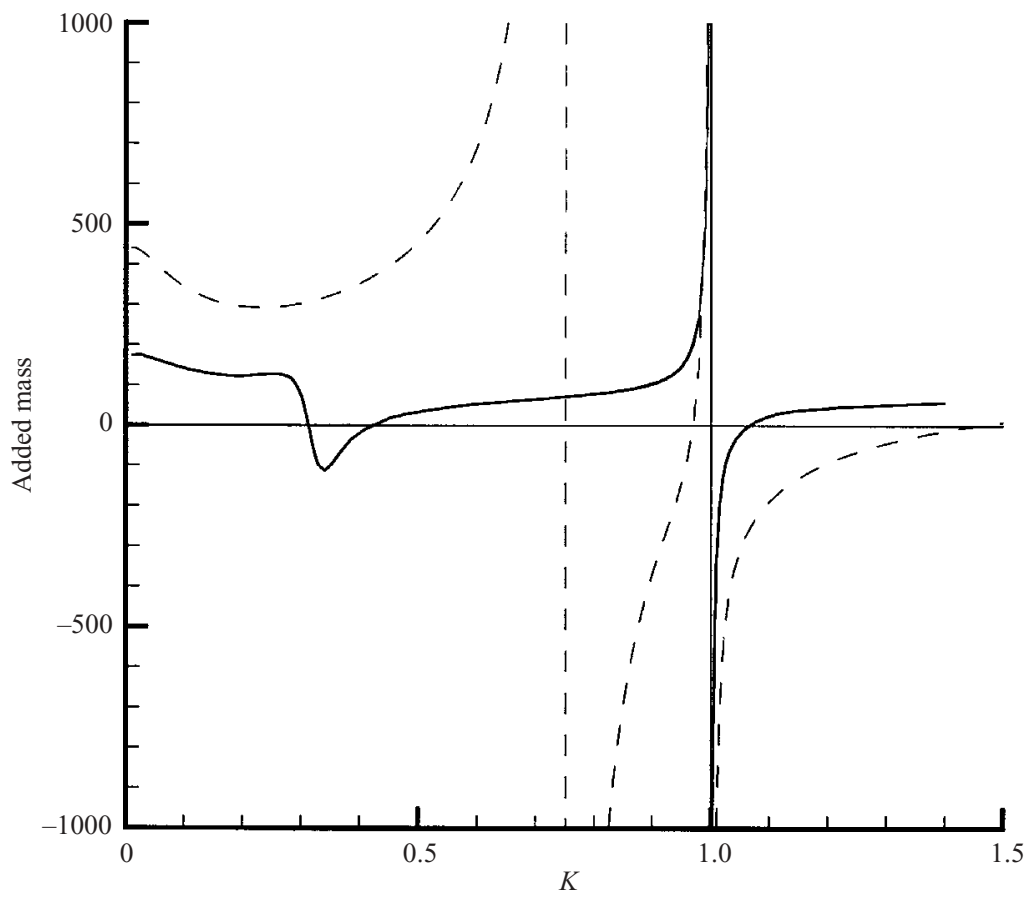

FiguRE 7. Heave added-mass coefficient of two axisymmetric structures generated by a ring source of radius $c=j_{0,2}$. The solid line is for a single 'outer' torus which encloses the ring source, with inner and outer waterline radii of 4.13 and 5.80 respectively. The dashed line is for the same outer structure, combined with an inner structure with waterline radii 1.00 and 3.56 .

As noted earlier in the discussion of figure 3, two classes of axisymmetric trapping structure may be formed from the flow field due to a ring source at $c=j_{0,2} / K$. These are concentric structures with two concentric elements of the type discussed in the previous paragraph, and structures in which only the outer element is included. The added mass for structures from each of these classes is shown in figure 7 with the ring-source radius chosen to be $c=j_{0,2}$. Without the inner element there is a trapped mode at $K=1$, which has a nodal circle on the interior free surface, and also a small region of negative added mass around $K=0.35$. In the vicinity of the latter feature the inner free surface oscillates as a pumping mode. When an inner structure is introduced the inner free surface is divided into two concentric rings. For the trapped mode these two free surfaces oscillate out of phase, while for the lower-frequency pumping mode they oscillate in phase. If the inner structure is increased in size from zero, the variation of the added mass around the pumping-mode frequency becomes more pronounced, as shown by the dashed line in figure 7 , and the wavenumber of the first sign change in the added mass first increases towards $K=1$ and then decreases.

Unlike the trapping mode, which has no radiation damping, the pumping mode is more conventional with radiated waves in the far field. However it is clear from the dashed curve in figure 7 that the radiation damping is extremely small for this structure. In an exact solution the peak amplitudes of the pumping-mode added mass would be finite, whereas the corresponding peaks of the trapping mode would be infinite. The finite numerical precision of these results precludes making such a distinction for this structure. 


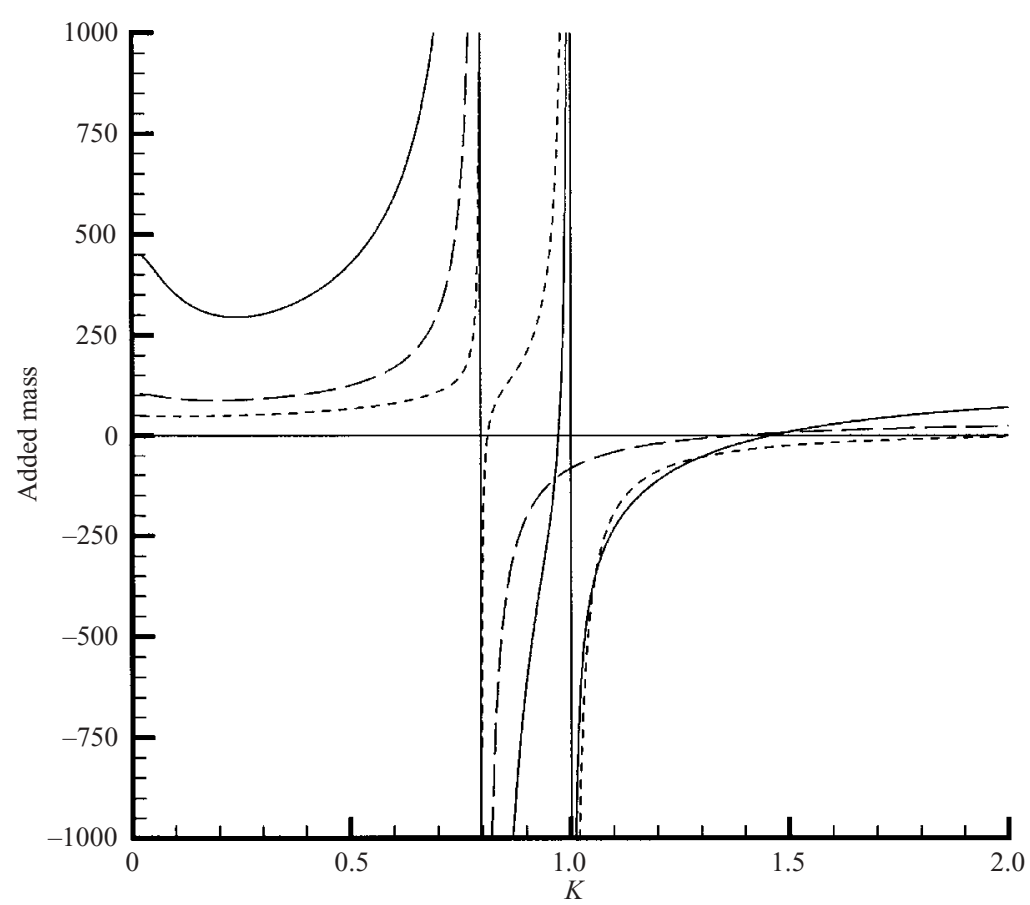

FIGURE 8. Added-mass coefficients for the concentric structure shown by dashed lines in figure 3; for the outer element the inner and outer waterline radii are 4.10 and 6.00 respectively, and for the inner element the radii are 0.89 and 3.62. The solid curve represents the added-mass coefficient for the complete structure. The long-dashed curve shows the heave force acting on the inner element alone with the outer element held fixed. The short-dashed curve shows the radial force for a radial motion of the inner element.

Further added-mass results for a structure formed from two concentric elements are shown in figure 8; here the structure is that shown by dashed lines in figure 3 for which both elements have a draught of 1.29. The structure is forced to move in three different modes: $(a)$ heave motion of the complete structure, $(b)$ heave motion of the inner element with the outer element held fixed, $(c)$ radial motion of the inner element with the outer element held fixed. The modes $(b)$ and $(c)$ are intended to be most effective in forcing the pumping and trapping modes, respectively, and the results confirm this. In each case there is a rapid change in added mass associated with the in-phase pumping mode highlighted by the results of figure 7 . However, singular behaviour at the trapped-mode wavenumber $K=1$ is apparent only for cases $(a)$ and (c). Remarkably, for case $(b)$, when the inner element alone is forced to heave, there is no anomalous behaviour around $K=1$. This can be explained as follows.

McIver (1997) shows that a necessary condition for the existence of the heave potential at the trapped-mode wavenumber $K_{\mathrm{T}}$ is that the trapped-mode potential $\phi$ must be orthogonal to the forcing from the heave motion; that is

$$
\iint_{S} \phi n_{z} \mathrm{~d} S=0
$$

where $S$ is the surface of those parts of the structure that are forced to heave, and $n_{z}$ is the vertical component of the inward normal to $S$. The integral in (5.1) can be evaluated by applying Green's theorem over the submerged interior of the structure 

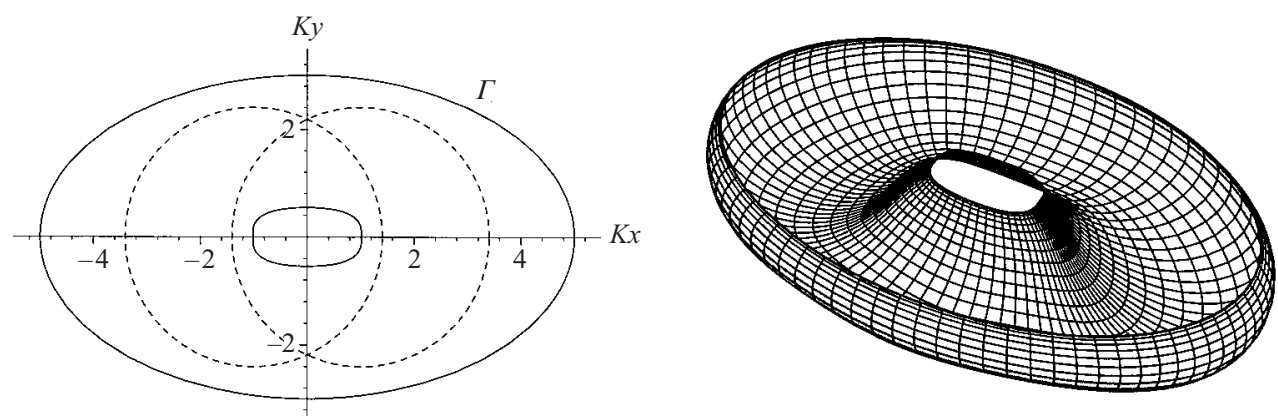

FIGURE 9. Inner and outer waterlines and the initial curve $\Gamma$ of a trapping structure corresponding to two displaced ring sources with equal strengths (the dashed lines show the positions of the ring sources), and a perspective view of the submerged surface.

to $\phi$ and $u=z-1 / K_{\mathrm{T}}$. Note that $u$ satisfies the same free-surface condition as $\phi$, and hence there is no contribution in Green's theorem from those parts of the interior free surface where $\phi$ is defined. If $S$, or part of $S$, contains the singularity then it is a simple matter to show that the integral in (5.1) is non-zero; in this case the heave potential does not exist and there is singular behaviour of the added mass. However, if that part of the structure around the singularity is held fixed then the application of Green's theorem shows that the integral in (5.1) is zero; hence the forcing is orthogonal to the trapped mode and the solution to the forcing problem exists at the trapped-mode frequency. Thus all integrated and local quantities, and in particular the added mass, are well-behaved at that frequency.

\section{Non-axisymmetric flow fields}

In this section examples are given of trapping structures that are obtained from the stream surfaces of non-axisymmetric flow fields formed by combining two ring sources in the free surface with centres at $(x, y)=( \pm \epsilon / K, 0), \epsilon>0$. Flow fields that do not involve wave radiation to infinity are obtained as long as for each ring the radius $c=j_{0, n} / K$ for some $n$. For each ring source, the value of $n$ and the strength need not be the same.

To illustrate the types of trapping structures that may be constructed, two examples are shown in figures 9 and 10. For the structure shown in figure 9 the two ring sources have the same radius, that corresponding to $n=1$, and equal strength. With this flow field stream surfaces can be generated by integrating from an initial curve $\Gamma$ that is entirely exterior to both ring sources. Not all choices of $\Gamma$ will lead to stream surfaces that exclude both singularities from the flow; however, for a given moderate value of $\epsilon$, it is not difficult to find choices of $\Gamma$ that lead to the generation of trapping structures with a single interior free surface. Here $\epsilon=1$ is used, so that the rings overlap, and $\Gamma$ is an ellipse with semi-major axis $5 / K$ and semi-minor axis $3 / K$ centred on the origin.

For the trapping structure in figure $10, \epsilon=0.5$ and rings of radius $j_{0,1} / K \approx$ $2.4048 / K$ and $j_{0,3} / K \approx 8.6537 / K$ are used so that the smaller ring lies entirely within the larger ring. Here the aim is to obtain a non-axisymmetric two-part structure enclosing two portions of the free surface in a similar way to the axisymmetric trapping structures constructed from concentric circular cylinders by Shipway \& Evans (2002) and the family of structures corresponding to figure 3. Thus separate 

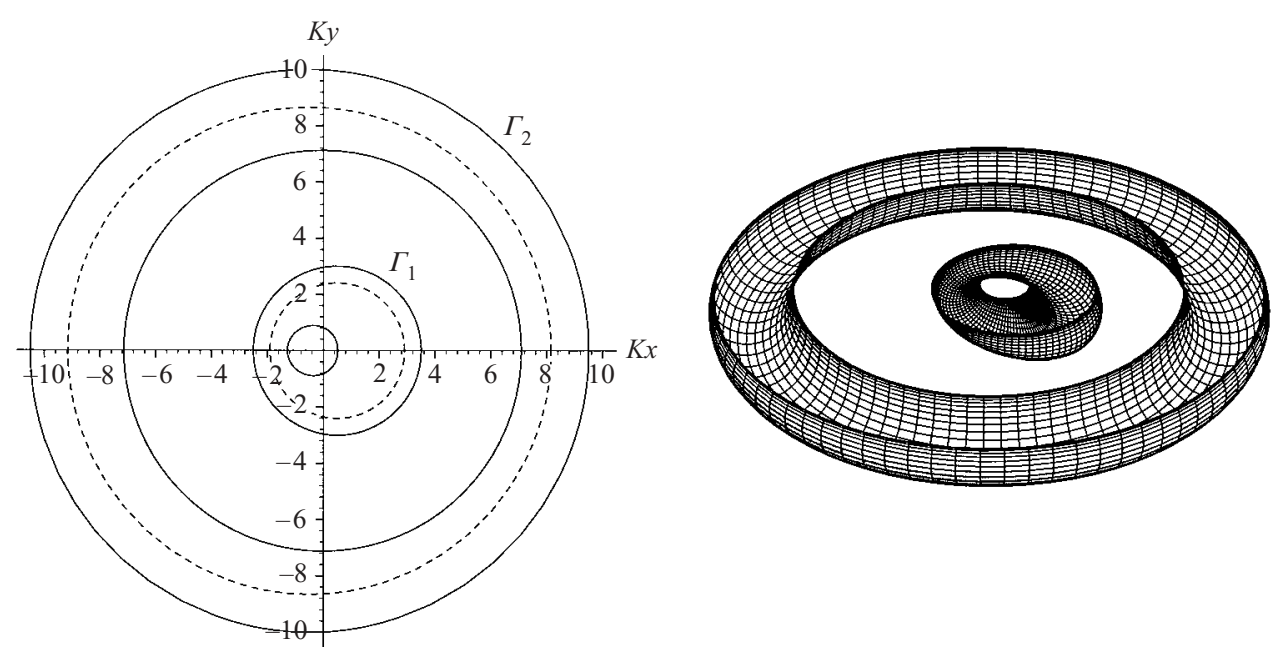

FIGURE 10. Inner and outer waterlines and the initial curves $\Gamma_{1}$ and $\Gamma_{2}$ of a trapping structure corresponding to two displaced ring sources with unequal strengths (the dashed lines show the positions of the ring sources), and a perspective view of the submerged surface.

stream surfaces are formed around each ring source by choosing two initial curves $\Gamma$. A concentric circle of radius $3 / K$ is used as the initial curve for the inner ring source, and for the outer ring source a concentric circle of radius $10 / K$ is used. To reduce the distortion of the inner stream surface, the strength of the outer ring source is one-tenth that of the inner ring source. For this particular example, where $n=3$ and the inner element is relatively small, one free-surface node exists in the outer moonpool; this is complementary to the lowest-order mode described by Shipway \& Evans (2002) where the node is in the inner moonpool.

The remaining constructions all give trapping structures, with two interior free surfaces that are 'separated' rather than quasi-concentric in the manner of the structure in figure 10. This is done by choosing the ring separation $\epsilon$ to be sufficiently large.

If $\epsilon$ significantly exceeds the radius of both ring sources then it is straightforward to obtain a trapping configuration which has two separate structures, each similar to the structure shown in figure 1. It is likely that trapped modes can be obtained in the presence of an array with any number $N$ of structures simply by constructing a flow field from $N$ widely separated ring sources.

Obtaining a single trapping structure with two separated interior free surfaces is more delicate. Constructions are described here for two cases when the oscillations in the interior free surfaces are $(a)$ in phase, and $(b)$ out of phase. For both examples there are two ring sources, each of radius $j_{0,1} / K$ and an offset $\epsilon=j_{0,1} / K$, so that the rings touch at the origin. The symmetry of the ring-source configuration will be exploited to obtain structures that are symmetric about the planes $x=0$ and $y=0$. In case $(a)$ both sources have strength +1 , and in case $(b)$ the strengths are \pm 1 .

The waterline and selected surface streamlines for a trapping structure of type $(a)$ are shown in figures 11 and 12. For a smooth doubly symmetric structure of this type, the symmetries of the flow field require the existence of a stagnation point $S$ where the $z$-axis intersects the surface of the structure. By construction of the flow field, the $x$ - and $y$-components of the velocity are zero everywhere on the $z$-axis for $z>0$. Numerical calculations show that there is a single point on the positive $z$-axis, at $z=z_{0} \approx 1.3827 / K$, where the $z$-component of the velocity is also zero and hence 


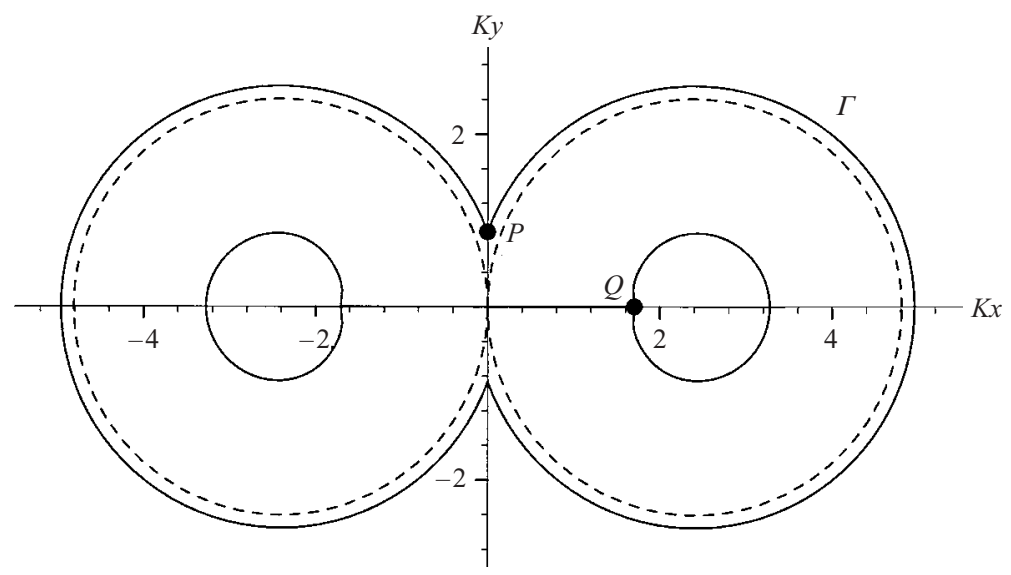

FIGURE 11. Inner and outer waterlines of a trapping structure corresponding to two displaced ring sources with equal strengths. The dashed lines show the positions of the ring sources.

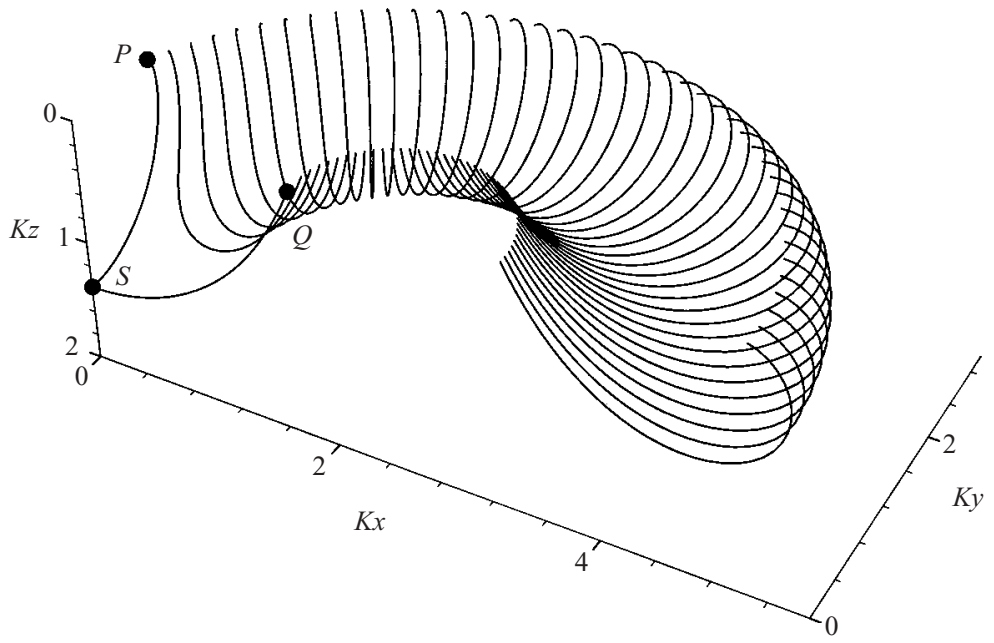

FIGURE 12. Selected surface streamlines for the trapping structure corresponding to the two displaced ring sources and initial curve $\Gamma$ shown in figure 11.

the surface of any trapping structure must contain this point. From this stagnation point it is straightforward to trace two 'dividing' streamlines. The first is in the plane $x=0$ and reaches the free surface in $y>0$ at a point $P$ that is exterior to the ring sources. The second is in the plane $y=0$ and reaches the free surface in $x>0$ at a point $Q$ that is interior to the ring source in $x \geqslant 0$. The construction is completed by tracing streamlines from an initial curve $\Gamma$ in $z=0$ which connects $P$ to the positive $x$-axis without intersecting either ring source. It is noteworthy that the surface of every structure constructed in this way will contain the streamlines from $S$ to $P$ and $Q$. For the example here, $\Gamma$ is a circular arc centred on $(x, y)=\left(j_{0,1} / K, 0\right)$ and the complete structure is obtained by reflection in $x=0$ and $y=0$.

The waterline and selected surface streamlines for a trapping structure of type $(b)$ are shown in figures 13 and 14. There appears to be more flexibility in this construction than for the type $(a)$ structure above as, for a given flow field, there are no streamlines that must lie on the surface of a trapping structure. The construction was begun by 


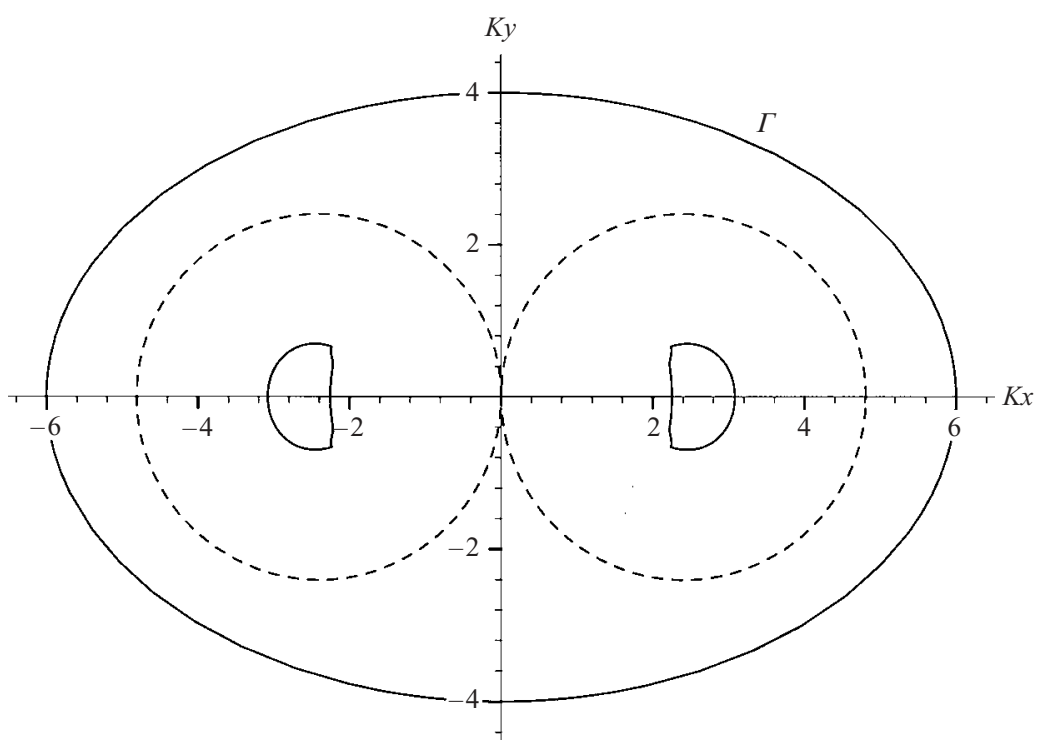

FIGURE 13. Waterline of a trapping structure corresponding to two displaced ring sources with strengths of equal magnitude and opposite sign. The dashed lines show the positions of the ring sources.

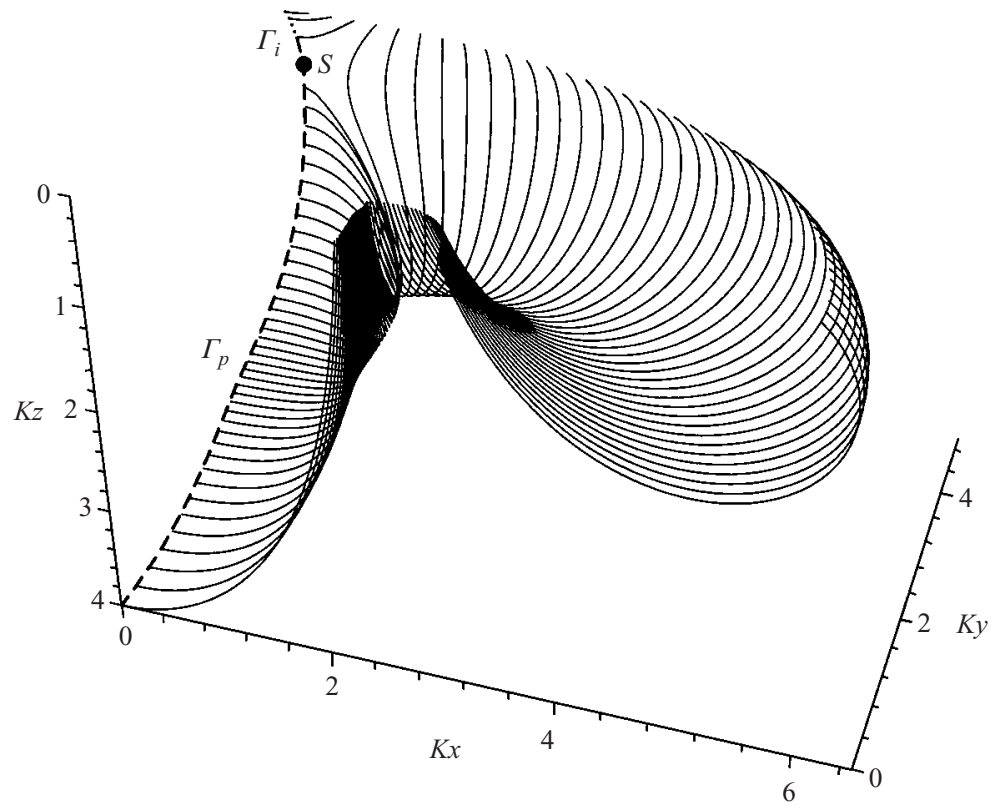

FIGURE 14. Selected surface streamlines for the trapping structure corresponding to the two displaced ring sources and initial curve $\Gamma$ shown in figure 13.

tracing streamlines from the initial curve $\Gamma$ shown in figure 13 ; here $\Gamma$ is a quarterellipse of semi-major axis $6 / K$ and semi-minor axis $4 / K$. Most of the streamlines emanating from $\Gamma$ go to an interior free surface in $x>0$, but some streamlines pass into $x<0$ beneath the free surface (see figure 14); the latter set of streamlines were traced using equation (3.1) and if extended would intersect the free surface on the 
reflection of $\Gamma$ in $x=0$. The curve where these streamlines intersect $x=0$ is shown by a dotted line in figure 14 and labelled $\Gamma_{i}$. To complete the construction $\Gamma_{i}$ was extended to form a curve that meets the $z$-axis and additional streamlines drawn from this curve. A difficulty is that there is a stagnation point $S$ on this curve; in fact the flow field has a stagnation line in the plane $x=0$ (which passes through the origin but otherwise lies in $z>0$ ).

To extend $\Gamma_{i}$ a polynomial was first fitted to the end points of the streamlines used to define $\Gamma_{i}$ (using streamlines closer to $S$ than those shown in figure 14). This polynomial was used to extrapolate from $\Gamma_{i}$ and to locate $S$ on the extension of $\Gamma_{i}$. Another curve $\Gamma_{p}$, shown by the dashed line in figure 14, was generated by fitting a cubic polynomial from the end of $\Gamma_{i}$, through $S$, to a point on the $z$-axis (chosen as $z=4 / K)$ such that $\Gamma_{p}$ intersects the $z$-axis at right angles. Further streamlines were then generated by integration from $\Gamma_{p}$ to the interior free surface within $x>0$; if these streamlines were continued into $x<0$ they would reach the second interior free surface. The dividing streamline from $S$ to the interior free surface is a line of discontinuity of slope on the surface of the trapping structure. The complete structure is obtained by reflection in $x=0$ and $y=0$.

\section{Discussion}

Special examples of three-dimensional floating bodies have been constructed which support trapped modes at specific frequencies. Unlike previous work (McIver 1996; McIver \& McIver 1997; Kuznetsov \& McIver 1997; Porter 2002; McIver \& Porter 2002) where the geometry of the body is either two-dimensional, or axisymmetric about the vertical axis, many of the present examples are fully three-dimensional. In some cases the bodies are constructed from non-axisymmetric stream surfaces, which nonetheless lie within axisymmetric velocity fields; in other cases both the body geometry and the global velocity field are non-axisymmetric.

Insofar as trapped modes do not radiate wave energy in the far field, it is logical to think of them as disturbances where the radiated waves cancel each other. Complete cancellation is simpler to envisage in two dimensions, or when the flow is axisymmetric about the vertical axis in three dimensions (or more generally in the latter case when only one Fourier component of the expansion in cylindrical coordinates is present). Thus it is significant to establish that fully three-dimensional trapped modes exist; in terms of a far-field expansion in cylindrical coordinates, all of the Fourier modes vanish.

The analyses of trapping structures fall into two categories: $(a)$ an indirect approach in which a wave-free singularity distribution is specified and then the corresponding stream surfaces computed, as in the present work; and $(b)$ a direct approach in which a class of structural geometries is specified, and parametric variations of its proportions are performed numerically to search for points in parameter space where non-trivial solutions of the homogeneous boundary-value problem exist (e.g. Porter 2002). The indirect approach has the advantage that the absence of radiated waves relies on exact analytic relations rather than on computations, although the trapping structure is constructed from the numerical computation of streamlines. (In the completely axisymmetric case considered by McIver \& McIver 1997, the existence of suitable streamlines was proved.)

Most of the trapping structures presented here have been analysed in the frequency domain using a three-dimensional radiation/diffraction code. The results in each case provide strong support for the existence of a trapping mode, although the 
finite precision of the computations precludes definite confirmation of pure trapping. Typical results are shown in figures 5,7 and 8. The structures with two moonpools exhibit resonant features not only at the trapped-mode wavenumber, but also at a secondary mode. These correspond to resonant motions of the moonpools with the same or opposite phase. The coupling of the two moonpools is similar to that described analytically by Miles (2002) for a simpler geometrical configuration. In most of the cases shown here the second (non-trapped) mode is associated with in-phase pumping, and occurs at a smaller wavenumber than the trapped mode. Conversely, for the structure shown in figures 11 and 12 where the two ring sources are in phase, the trapped-mode wavenumber is less than the secondary pumping mode; this is explained by the fact that the trapping mode is symmetric about the plane $x=0$, whereas the secondary mode is antisymmetric.

When the existence of trapping structures was first discovered (McIver 1996), the importance was primarily from the theoretical standpoint in establishing that there are body shapes and frequencies where the solutions of the linearized radiation and diffraction problems are either non-unique, or do not exist. From the standpoint of practical applications in offshore engineering, various types of floating structures are constructed with one or more 'moonpools', interior chambers of fluid with free surfaces which are open to the exterior domain at the bottom. It is well known that the fluid motion in moonpools is magnified at resonant wave periods, including a large-amplitude vertical response at the piston-like 'pumping' mode as well as 'sloshing' modes which are analogous to standing waves within the moonpool. In general these motions are weakly damped by energy radiation in the exterior domain (in practice viscous damping may be more important). The discovery of trapped modes for particular structures with moonpools provides further insight into these resonances, and demonstrates that in certain cases there is no wave damping at all. All the examples of trapping structures described in this paper have moonpools.

It seems likely that many other types of trapping structure exist, and the examples that have been derived so far probably represent only the 'tip of the iceberg'. Possible classes of trapping structures for which no examples are known, if indeed they exist at all, include isolated floating structures without an internal free surface, and structures that support trapped modes at two different frequencies. (One of the referees has pointed out that in the oral presentation of Shipway \& Evans 2002, the authors reported the construction of a trapping structure that supported two trapped modes at distinct frequencies.)

This work was funded in part by grant number GR/M30937 awarded by the UK Engineering and Physical Sciences Research Council. Substantial assistance with the computations shown in figures 7 and 8 has been provided by Dr John Letcher of AeroHydro, Inc., and Dr Chang-Ho Lee of WAMIT, Inc.

\section{REFERENCES}

Bourne, D. E. \& Kendall, P. C. 1977 Vector Analysis and Cartesian Tensors, 2nd Edn. Van Nostrand Reinhold.

Callan, M., Linton, C. M. \& Evans, D. V. 1991 Trapped modes in two-dimensional waveguides. J. Fluid Mech. 229, 51-64.

Courant, R. \& Hilbert, D. 1962 Methods of Mathematical Physics: Vol. II. John Wiley \& Sons.

Evans, D. V. \& PoRTER, R. 1998 Trapped modes embedded in the continuous spectrum. Q. J. Mech. Appl. Maths 52, 263-274.

John, F. 1950 On the motion of floating bodies, II. Commun. Pure Appl. Maths 3, 45-101. 
Kuznetsov, N. \& McIver, P. 1997 On uniqueness and trapped modes in the water-wave problem for a surface-piercing axisymmetric body. Q. J. Mech. Appl. Maths 50, 565-580.

LeE, C.-H. \& Newman, J. N. 2001 Solution of radiation problems with exact geometry. In Proc. 16th Intl Workshop on Water Waves and Floating Bodies, Hiroshima, Japan (ed. K. Mori \& H. Hidetsugu), pp. 93-96.

MCIvER, M. 1996 An example of non-uniqueness in the two-dimensional linear water wave problem. J. Fluid Mech. 315, 257-266.

MCIver, M. 1997 Resonance in the unbounded water wave problem. In Proc. 12th Intl Workshop on Water Waves and Floating Bodies, Carry-le-Rouet, France (ed. B. Molin), pp. 177-180.

MCIver, P. \& MCIVER, M. 1997 Trapped modes in an axisymmetric water-wave problem. Q. J. Mech. Appl. Maths 50, 165-178.

McIver, M. \& Porter, R. 2002 Trapping of waves by a submerged elliptical torus. J. Fluid Mech. 456, 277-293.

MiLES, J. 2002 On slow oscillations in coupled wells. J. Fluid Mech. 455, $283-287$.

Newman, J. N. 1999 Radiation and diffraction analysis of the McIver toroid. J. Engng Maths 35, $135-147$.

PorTER, R. 2002 Trapping of water waves by pairs of submerged cylinders. Proc. R. Soc. Lond. A 458, 607-624.

PozRIKIDIs, C. 1997 Introduction to Theoretical and Computational Fluid Dynamics. Oxford University Press.

ShipwaY, B. J. \& Evans, D. V. 2002 Wave trapping by axisymmetric concentric cylinders. In Proc. 21st Intl Conf. on Offshore Mechanics and Arctic Engineering, Oslo, Norway, June 23-28, 2002. ASME. 